\title{
Wear Protection Without Surface Modification Using a Synergistic Mixture of Molecular Brushes and Linear Polymers
}

\author{
Jimmy Faivre ${ }^{1,3}$, Buddha R. Shrestha ${ }^{1}$, Joanna Burdynska ${ }^{2}$, Guojun Xie, ${ }^{2}$ Florina Moldovan ${ }^{3}$, \\ Thierry Delair ${ }^{4}$,Stéphane Benayoun, ${ }^{5}$ Laurent David ${ }^{4}$, Krzysztof Matyjaszewski $^{2 *}$, Xavier \\ Banquy $^{1 *}$ \\ ${ }^{1}$ Canadian Research Chair in Bioinspired materials, Faculty of Pharmacy, Université de \\ Montréal, Montréal, Qc, Canada \\ ${ }^{2}$ Center for Macromolecular Engineering, Department of Chemistry, Carnegie Mellon \\ University, Pittsburgh, PA, USA \\ ${ }^{3}$ CHU Sainte Justine Research Center and Department of Stomatology, Faculty of Dentistry \\ ${ }^{4}$ Université de Lyon, Université Claude Bernard Lyon 1, CNRS, Ingénierie des Matériaux \\ Polymères (IMP-UMR 5223), 15 Boulevard Latarjet, 69622 Villeurbanne Cedex, France \\ ${ }^{5}$ Laboratoire de Tribologie et Dynamique des Systèmes, UMR 5513 CNRS, Ecole Centrale de \\ Lyon, 36 Avenue Guy de Collongue, 69134 Ecully Cedex, France
}

Corresponding authors: $\underline{\mathrm{km} 3 \mathrm{~b} @ \text { andrew.cmu.edu; }}$ xavier.banquy@umontreal.ca

\begin{abstract}
.
We describe the design of lubricating and wear protecting fluids based on mixtures of bottlebrushes (BB) and linear polymers solutions. To illustrate this concept we used hyaluronic acid (HA) - a naturally occurring linear polyelectrolyte, and a water soluble synthetic BB polymer. Individually, these two polymers exhibit poor wear protecting capabilities compared to saline solutions. Mixture of the two polymers in pure water or in saline allows to drastically increase wear protection of surfaces over a wide range of shearing conditions. We demonstrate that this synergy between the BB and HA polymers emerges from a strong cohesion between the two components forming the boundary film due to entanglements between both polymers. We show that this concept can be applied to other types of linear polymers and surfaces and is independent of the chemical and mechanical properties of the surfaces.
\end{abstract}

\section{Keywords.}

Biolubrication, wear resistance, bottle-brush polymer, surface forces, hyaluronic acid 
With the ever-increasing need of more efficient and long lasting machinery and devices, certain issues such as control of wear and fatigue of machine parts have become extremely challenging. ${ }^{1}$ The design of lubricating fluids able to protect surfaces against wear and high friction has been one the several tools used by engineers to improve machines' life time. ${ }^{2}$

It is generally assumed that damage caused during sliding, commonly known as "abrasive friction", is due to a high friction force and, therefore, a large coefficient of friction. Accordingly, to prevent surface damage or wear one should aim to reduce the coefficient of friction, which has been the traditional focus of basic research into many bio and nonbiolubrication systems. However, many biological and non-biological systems (especially involving soft polymeric surfaces) exhibit very complex behavior where the coefficient of friction and wear (abrasion) are not simply related, and sometimes even have an inverse relationship. Therefore, other factors, such as the surface structure, the lubricant distribution and conformation, and the lubricant-surface interaction are certainly more important than the coefficient of friction in determining the onset of wear.

Recent advances have suggested that using inspiration from nature, lubricating fluids or coatings could provide enhanced wear protection without any loss of lubrication. ${ }^{3,4}$ The exceptional wear resistance and unparalleled lubricating properties of articular joints have motivated a lot of research aimed to unravel the molecular mechanism at the origin of joints tribological properties. ${ }^{5,6}$ Several design strategies aimed to enhance wear protection have emerged involving polymer brushes either in their molecular form as BB polymers, ${ }^{7-10}$ or grafted on surfaces as polymer brush coatings. ${ }^{11-15}$ Polymer brushes are known to provide excellent lubrication to surfaces via low interpenetration of polymer chains under compression. ${ }^{16,17}$ Other mechanisms involving linear polymers mechanically trapped at the interface have also been suggested as a mechanism used by nature to maintain high wear resistance even under high compressive stress. All these strategies require the lubricating or wear protecting molecules to be strongly anchored to the surfaces in order to avoid close contact between the surfaces. Strong anchoring of molecules on surfaces requires a good knowledge of the chemistry and structure of the surface which complicates dramatically the translation of these technologies towards industrial settings.

In this study we show that it is possible to design lubricating fluids able to provide excellent wear protection without any chemical modification of the surfaces. The fluids use two components, BB polymers shown in Figure 1A containing zwitterionic pendant chains synthesized by atom transfer radical polymerization, ATRP ${ }^{18,19}$ (see SI for detailed synthesis procedure) and a natural linear polymer, sodium hyaluronate (HA, Figure 1B). Both components are soluble in pure water or saline conditions. 

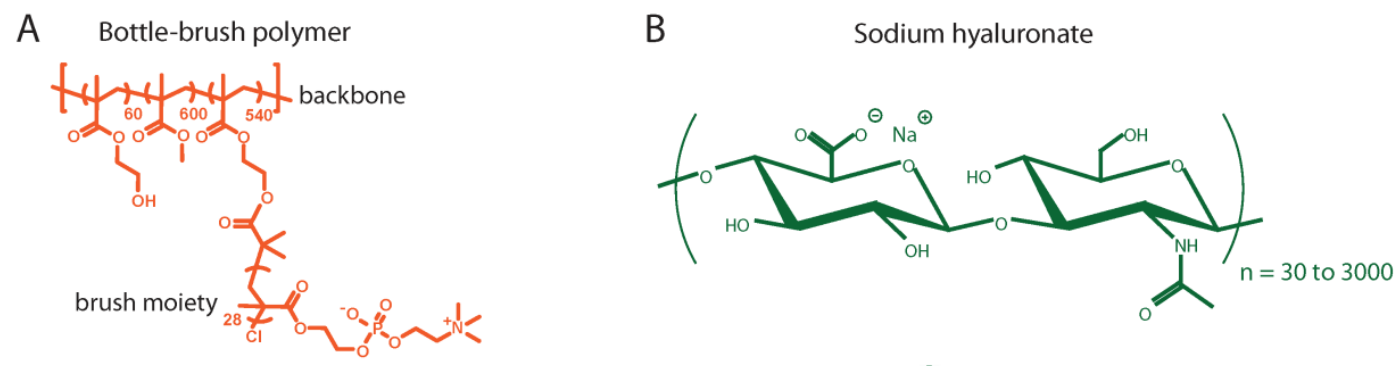

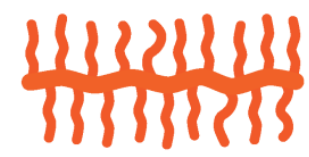

C

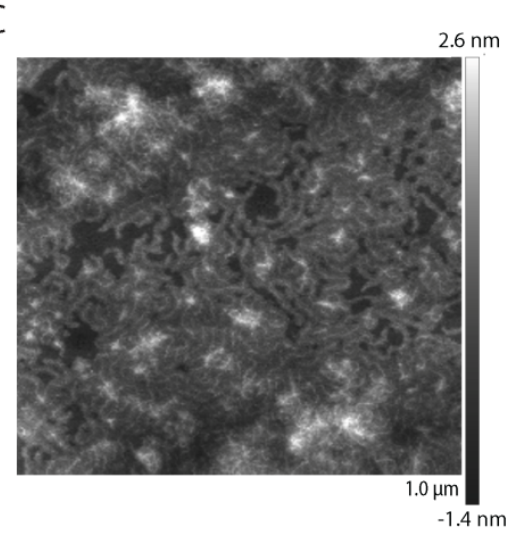

D

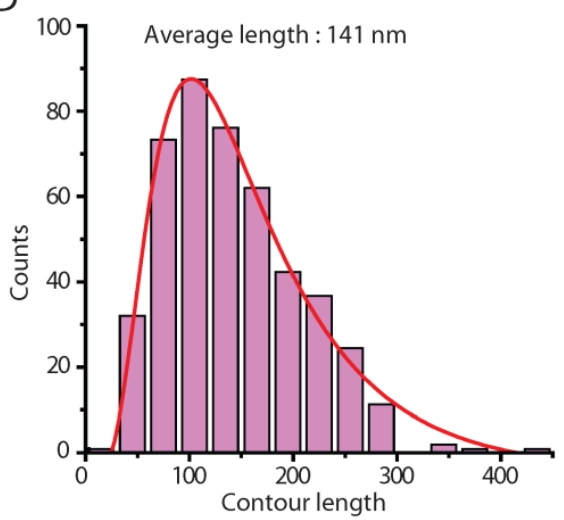

$\mathrm{E}$

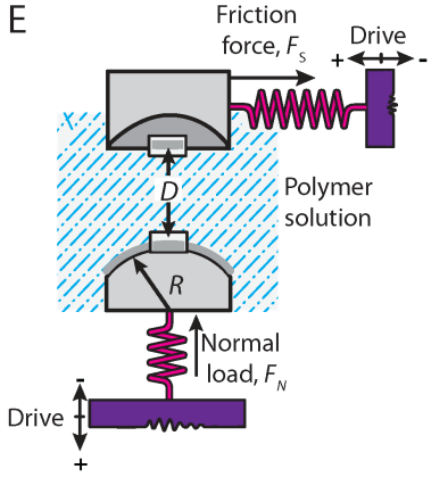

Figure 1. (A) Schematic representation of the BB polymer and (B) the sodium hyaluronate polymers used in the present study; (C) Atomic force microscope image in air of the BB polymer deposited on mica and (D) its contour length distribution; (E) Mechanical equivalent of the experimental set up of the Surface Forces Apparatus in the used configuration.

We used the surface force apparatus (SFA) to characterize the wear protection capacity and the lubricating properties of the fluids (see Figure 1E and materials and methods for procedures). The SFA allows measuring frictional forces over a wide range of pressure and sliding speeds while monitoring the separation distance between the surfaces at $\pm 0.5 \AA$ resolution as well as the shape of the contact. Muscovite mica is the substrate of choice in SFA experiments mostly because of its optical transparency and atomic flatness. In our particular study, mica was used due to its extreme propensity to suffer damage under moderate shearing conditions in water and saline conditions which makes it the perfect substrate to test wear protection.

\section{Results and Discussion.}

We first measured the normal interaction forces, $F_{\mathrm{N}}$, between two facing mica surfaces of curvature $R$, in presence of the different components of the lubricating fluids, first individually and then mixed together. To cover a wide range of conditions, we tested different HA molecular 
weights, $M_{\mathrm{w}}$, in pure water and in phosphate buffer salines (PBS $150 \mathrm{mM}$ for low ionic strength, and $1500 \mathrm{mM} \mathrm{NaCl}$ for high ionic strength, both at $\mathrm{pH}=7.4$, see Figure 2). The interaction forces were recorded as a function of the separation distance, $D$, between the surfaces starting from several hundreds of nanometers (zero interaction regime) down to a few angstroms (strong interaction regime) in order to capture the full interaction force profile (force law) of the system.

In Figures $2 \mathrm{~A}$ and $\mathrm{S} 3$ are shown the force profiles measured in HA solutions in pure water and in saline buffers. HA being negatively charged under all tested conditions, it is expected to adsorb as a random coil on negatively charged mica surfaces through the formation of hydrogen bonds. As shown in Figure 2A, force profiles present two distinctive trends, depending on the ionic strength of the medium. In pure water, the onset of the interaction forces was located between 20 and $30 \mathrm{~nm}$ independently of the molecular weight of HA (see Figure S3). Such weak dependence on the molecular weight suggests that during the time window of the experiment, only low molecular weight chains could adsorb on the surfaces (a case similar to the Vroman effect). Low molecular weight molecules are expected to adsorb first on mica surfaces, since they are more mobile. Later, larger molecules which have higher affinity for the surface, are expected to displace them. In saline, the interaction forces exhibited shorter range forces starting between 5 to $10 \mathrm{~nm}$. These short range interaction forces systematically presented periodic instabilities indicating the presence of a layered structure at the surfaces (see inset of Figure 2A). The characteristic size $\Delta D$ of these instabilities was $\Delta D=0.2-0.3 \mathrm{~nm}$ in agreement with the size of a water molecule. Adhesive forces were systematically measured upon separation of the surfaces independently of the medium (pure water or saline). 

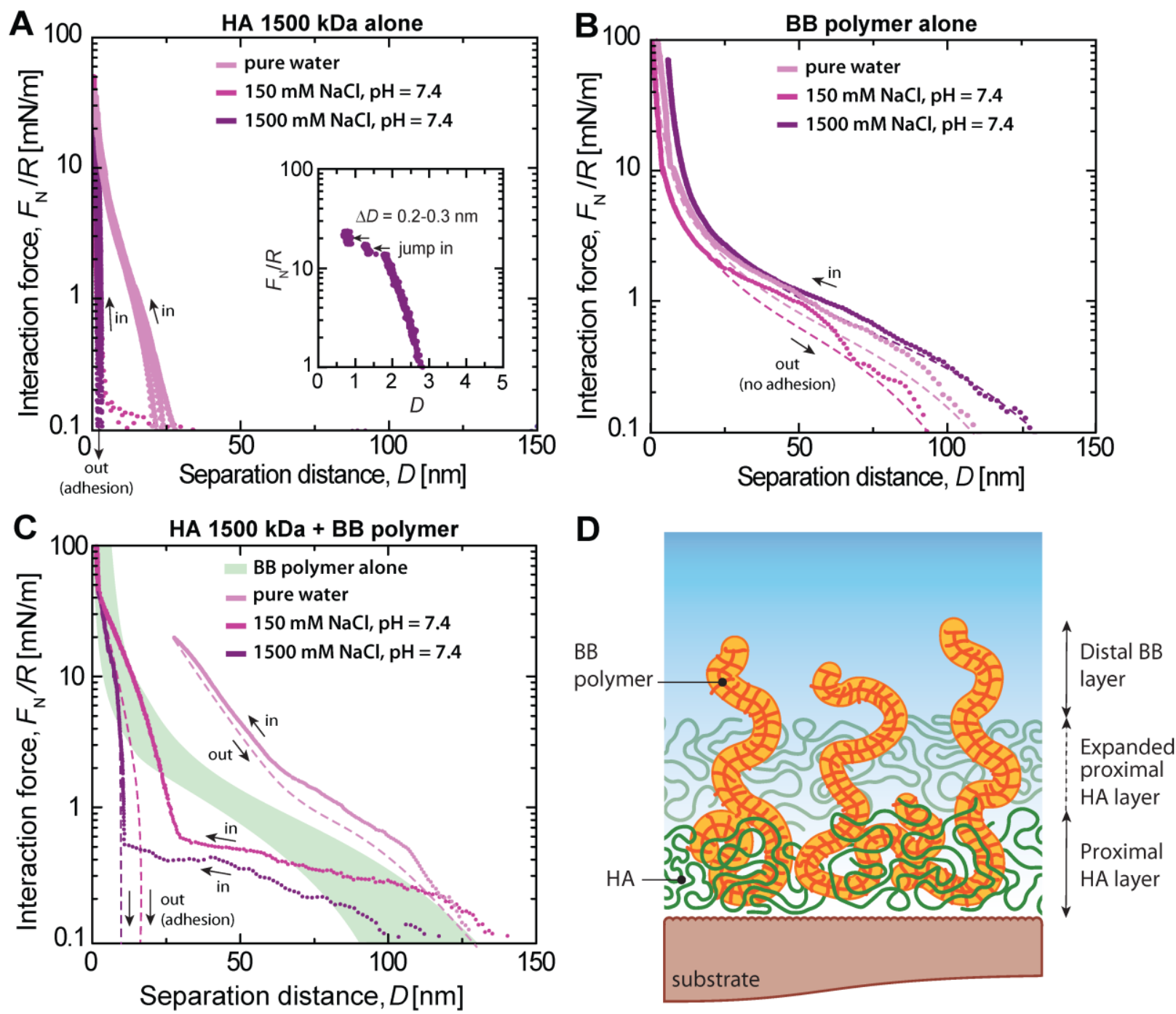

Figure 2. Interaction force profiles measured across (A) HA solution $(1 \mathrm{mg} / \mathrm{mL})$ at increasing ionic strength. Inset is an expanded view of the force profile at $1500 \mathrm{mM} \mathrm{NaCl}$ showing characteristic step-like instabilities in the interaction forces indicating the presence of a layered structure in the confined space; (B) BB polymer solution $(0.1 \mathrm{mg} / \mathrm{mL})$ at increasing ionic strength; (C) Mixture of $\mathrm{BB}$ polymer and $\mathrm{HA} 1500 \mathrm{kDa}$ at different ionic strength. (D) Schematic representation of the interfacial polymer layer in presence of $\mathrm{HA}$ and BB polymers.

These observations demonstrate that under the present experimental conditions, HA does not strongly bound to the mica surfaces in saline due to the presence of a 2 to $3 \mathrm{~nm}$ thick hydration layer strongly interacting with the surface, while in pure water, the polymer can adsorb strongly and form a stable soft layer.

Interaction forces in presence of the BB polymer alone were strikingly different from HA polymer alone (see Figure 2B). Repulsive forces were measured on approach and separation of the surfaces, independently of the ionic strength of the medium. More interestingly, the force 
profiles were insensitive to the ionic strength of the medium. Interestingly, the conformation of linear polymer chains of MPC has been reported to be insensitive to the ionic strength in solution $^{20}$, and at surfaces. ${ }^{21}$ The onset of the interaction forces (determined at $F_{\mathrm{N}} / R=0.01$ $\mathrm{mN} / \mathrm{m}$ ) was found to vary between $100 \mathrm{~nm}$ and $125 \mathrm{~nm}$, independently of the medium ionic strength (Figure 2B). Given that the contour length of the polymer, assessed by AFM imaging in air (see Figure 1D), is $\sim 140 \mathrm{~nm}$, a significant part of the BB polymer is expected to be extending towards the medium.

Interaction forces under high confinement $(D<10 \mathrm{~nm})$ did not present any layering transition or any evidence of hydration forces which confirms that the BB polymer interacts strongly with the hydrated surface layer, strongly enough to displace the water molecules present at the surface. Such observations echoes some reports showing that charged amine head groups adjacent to $\mathrm{H}-$ bonding donors groups can efficiently remove bound water from a hydrated surface and facilitate $\mathrm{H}$ - bonding. ${ }^{22,23}$ No adhesive forces were measured upon separation of the surfaces.

Interaction forces across $\mathrm{HA}-\mathrm{BB}$ polymer mixtures in saline presented similar features to $\mathrm{BB}$ polymer alone (Fig 2C and S4). Exception made of the mixture containing $\mathrm{HA} 10 \mathrm{kDa}$, the onset of the interaction forces measured for the different polymer mixtures ranged between $120 \mathrm{~nm}$ and $180 \mathrm{~nm}$ depending on the medium, which is similar to the onset measured with the BB polymer alone and at least twice the value measured for any of the tested HA alone solutions. Below a separation distance $D \approx 50 \mathrm{~nm}$, a steep increase in the interaction forces was systematically observed suggesting the presence of a dense/stiff layer of polymer at the surfaces. The thickness of this dense (proximal) polymer layer, was found to be highly sensitive to the ionic strength of the medium. Based on the force profiles, the thickness of the proximal layer can vary from approximately $20 \mathrm{~nm}$ at $150 \mathrm{mM} \mathrm{NaCl}$ to $5 \mathrm{~nm}$ at $1500 \mathrm{mM} \mathrm{NaCl}$. No adhesive forces were measured if the surfaces were separated at D> $50 \mathrm{~nm}$ (in the distal region of the interaction profile) while weak adhesive forces were systematically observed when separating the surfaces at $D<50 \mathrm{~nm}$. These observations confirmed that the proximal layer contains mainly HA.

These force profiles demonstrate that the polymer mixtures form an interpenetrated layered thin film as represented in Figure 2D. The proximal layer of such film contains most of the HA molecules adsorbed at the surface and portions of BB polymer chains while the distal layer is composed solely of BB polymer molecules extending in the medium.

In pure water, the force profiles of the different mixtures did not exhibit any marked transition between the HA-rich proximal layer and the BB polymer distal layer. Instead, the force profiles show a continuous increase, consistent with an extended proximal layer fully overlapping with the distal layer.

After measuring the normal interaction forces in the different media, we characterized the tribological properties of the different polymer mixtures. In a first series of experiments, we measured the friction force, $F_{\mathrm{S}}$, as a function of the applied normal force $F_{\mathrm{N}}$ (Figure 3). For all 
the tested conditions, the friction force, $F_{\mathrm{S}}$, was found to increase linearly with $F_{\mathrm{N}}$ until damage of the surfaces occurred (see Figure 3A). We therefore defined the friction coefficient of our system as $\mu=F_{\mathrm{S}} / F_{\mathrm{N}}$. In saline only (no polymer added), frictional forces were very weak (not shown), giving a friction coefficient of $\mu=0.002 \pm 0.001$ (Figure 3B), in good agreement with previous reports. ${ }^{24}$ Using optical interferometry, ${ }^{25}$ we measured the critical pressure, $P^{*}$, at which the onset of surface damage was triggered. Onset of damage appeared as sudden cracks formation and propagation along the direction of shearing. We found a value of $P^{*}=0.73 \pm 0.03$ MPa for both saline conditions. We also found that $\mu$ increased two orders of magnitude after damage occurred and ranged between 0.2 and 0.7 , as shown in Figure 3B. On the other hand, in pure water, damage of the surfaces occurred almost immediately after a few shearing cycles indicating that $P^{*} \approx 0 \mathrm{MPa}$. The present results echo recent studies demonstrating that, in saline medium, surface adsorbed ions facilitate the formation of a lubricating water layer able to sustain a significant amount of normal pressure under shear and therefore protecting the surfaces from damage. $^{26,27}$
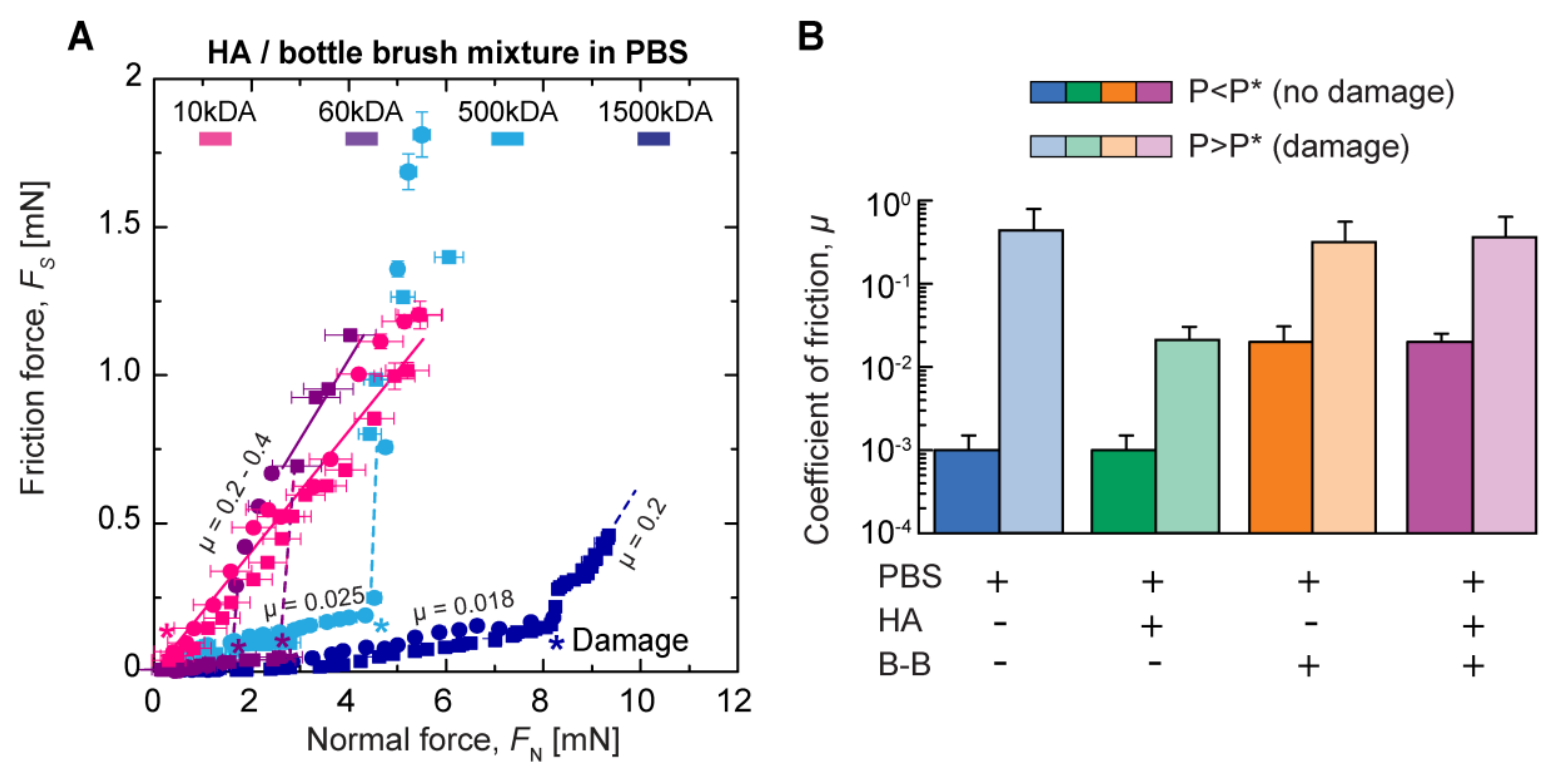

Figure 3. (A) Tribological testing of the BB $(0.1 \mathrm{mg} / \mathrm{mL})$ and $\mathrm{HA}(1 \mathrm{mg} / \mathrm{mL})$ polymer mixtures in PBS (150 mM NaCl) performed at a sliding speed of $3 \mu \mathrm{m} / \mathrm{s}$. (B) Measured friction coefficients in saline $(150 \mathrm{mM}$ and $1500 \mathrm{mM} \mathrm{NaCl})$ before and after damage in presence of $\mathrm{BB}$ and HA polymers, alone and mixed together. Onset of damage is indicated by * symbol.

In presence of HA, the measured values of $\mu$ in saline before damage $\left(P<P^{*}\right)$ were found to be independent of the molecular weight of the polymer (Figures $3 \mathrm{~B}$ ) and close to the values found in saline only $\left(\mu \approx 10^{-3}\right)$. Measurements of the thickness and refractive index of the confined film before damage shows that the contact area is quickly depleted of polymer leaving only adsorbed ions and water molecules at the interface (see Figure S5). The value of $P^{*}$ for HA was 
$P^{*} \approx 0.7 \mathrm{MPa}$ independently of its molecular weight, which is identical to the value encountered in saline only and consistent with the previous observation of HA being depleted from the contact before damage occurs. In pure water, HA solutions demonstrated very poor stability and systematically lead to the formation of polymer aggregates in the shearing contact. These polymer aggregates lead to focal pressure increase throughout the contact area and eventually triggered crack formation. As a consequence, the measured value of $P^{*} \approx 0 \mathrm{MPa}$ even though $\mu$ $=0.02$ for all the $M_{\mathrm{w}}$ tested after damage occurred.

Frictional properties of the BB polymer alone in pure water and saline were drastically different from HA or saline alone (Figure 3B). The measured friction coefficient $\mu$ before damage was one order of magnitude higher than HA or saline alone and was equal to $\mu=0.03 \pm 0.01$, independently of the ionic strength of the medium. This result is consistent with the previous observation from the normal force profiles showing that BB polymer adsorption and conformation on mica was independent of the ionic strength. In saline and pure water, the measured values of $P^{*}$ were $0.25 \pm 0.02$ and $0.56 \pm 0.04$ respectively, which is lower than HA and saline alone under similar conditions.

As shown in Figure 3A and B, mixing HA and the BB polymer did not improve significantly the lubricating properties of the surfaces. The measured friction coefficient of the different mixtures before damage $\left(P<P^{*}\right)$ was independent of HA molecular weight and equal to $\mu=0.02 \pm 0.01$, independently of the medium's ionic strength. This observation suggests that the friction coefficient of the mixture is solely controlled by the presence of the BB polymer when $P<P^{*}$.

Most interestingly, the value of $P^{*}$, which relates to the wear protection capacity of the polymer mixture, was highly sensitive to HA molecular weight. As can be seen in Figure 4A, for mixtures of final concentration $100 \mu \mathrm{g} / \mathrm{mL}$ of BB polymer and $1 \mathrm{mg} / \mathrm{mL}$ of HA (1:10 mass ratio), $P^{*}$ increased significantly with HA molecular weight as $P^{*} \propto \log \left(M_{\mathrm{w}}\right)$. In pure water, the HA - BB polymer mixture lead systematically to a significant increase in wear protection, especially at high HA molecular weight, with $\mathrm{P}^{*}$ increasing from $0 \mathrm{MPa}$ in absence of $\mathrm{BB}$ polymer to 3.2 $\mathrm{MPa}$ in presence of BB polymer. A similar trend was observed in saline solutions, with a two fold increase of $P^{*}$ at the highest HA molecular weight in presence of the BB polymer. 

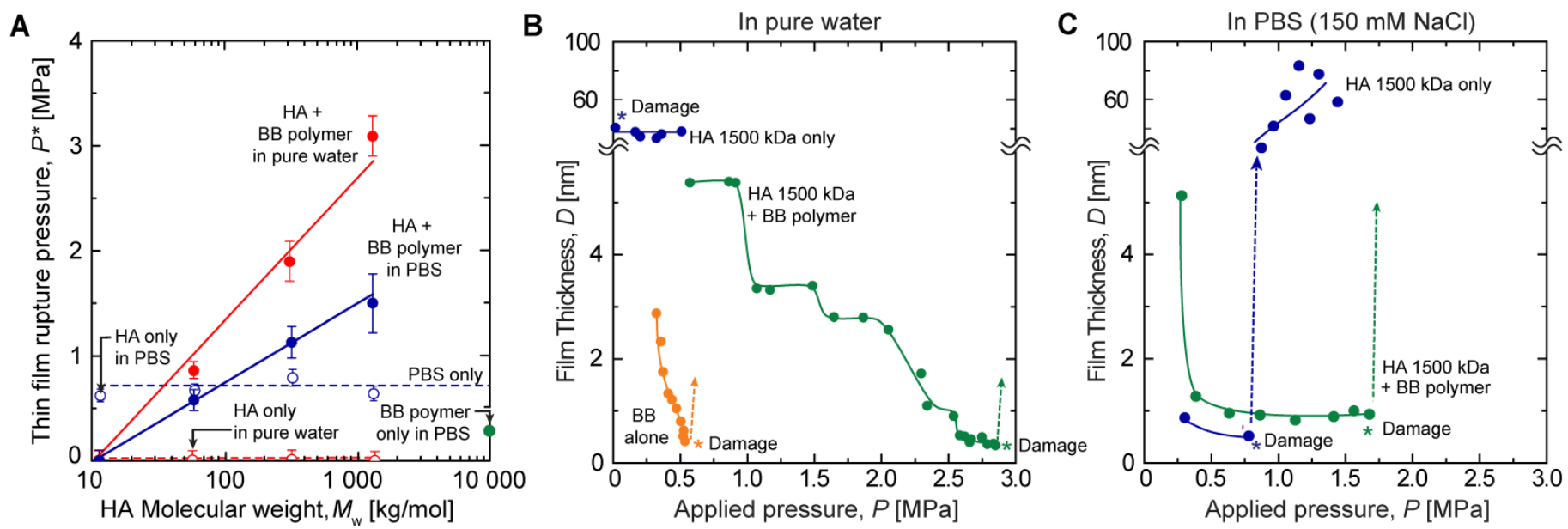

Figure 4. Synergistic enhancement of wear protection using HA and BB polymer mixtures. (A) The thin film rupture pressure $P^{*}$ was measured in presence of $1 \mathrm{mg} / \mathrm{mL}$ HA solution mixed with a $0.1 \mathrm{mg} / \mathrm{mL} \mathrm{BB}$ polymer solution at a sliding speed of $3 \mu \mathrm{m} / \mathrm{s}$. The value of $P^{*}$ for each of the component alone was below the value of pure PBS independently of HA molecular weight, while the mixtures presented significantly higher value of $P^{*}$, especially at high HA $M_{\mathrm{w}}$. (B-C) Measurements of the thin film thickness during shear in pure water and in PBS shows that the polymer mixtures are able to sustain significantly more pressure compared to the polymers alone and in a $M_{\mathrm{w}}$-dependent manner.

To obtain more insights into the mechanism underlying such phenomenon, we monitored the evolution with shearing time of the film thickness under different shearing conditions. Figure 4B shows that, as the normal pressure $P$ is increased, the film thickness, $D$, increases dramatically when the medium contains HA only in pure water, indicating the immediate aggregation of the polymer and the triggering of surface wear. In saline (Figure 4C and S5), the data show that HA is quickly depleted from the contact leading to a rapid decrease of $D$ down to $0.5 \mathrm{~nm}$ before damage occurs at $P=P^{*}$.

In presence of $\mathrm{BB}$ polymers alone, the film thickness at $P=P^{*}$ was $1 \mathrm{~nm}$ for both saline conditions which is thicker than the previously mentioned value obtained for HA solutions. Such high value of the film thickness indicates that BB polymer chains are still present in the contact at the onset of wear. Similar observations were confirmed with the different polymer mixtures, although the values of $P^{*}$ were significantly higher than $\mathrm{BB}$ or $\mathrm{HA}$ alone (Figures $4 \mathrm{~B}$ and $\mathrm{C}$ ). The significant increase of $P^{*}$ in the case of the polymer mixtures correlates with the higher film thickness at the onset of damage which indicates the existence of strong intermolecular interactions between HA and the BB polymer. Such interactions maintain a strong cohesion between the different polymer chains under shearing conditions and allows the confined film to sustain significantly more normal pressure.

To elucidate the nature of the interactions responsible of such strong intermolecular cohesion, we performed a series of isothermal titration calorimetry experiments (ITC, see Figure S6). No 
thermal signature was measured during mixing of the polymers indicating that no detectable interaction (electrostatic or hydrophobic) exists between the two polymers. Therefore, the important role played by HA molecular weight in tuning the cohesive strength of the film demonstrate that chain entanglements are the main factor responsible for the polymer film cohesion (Figure 5).
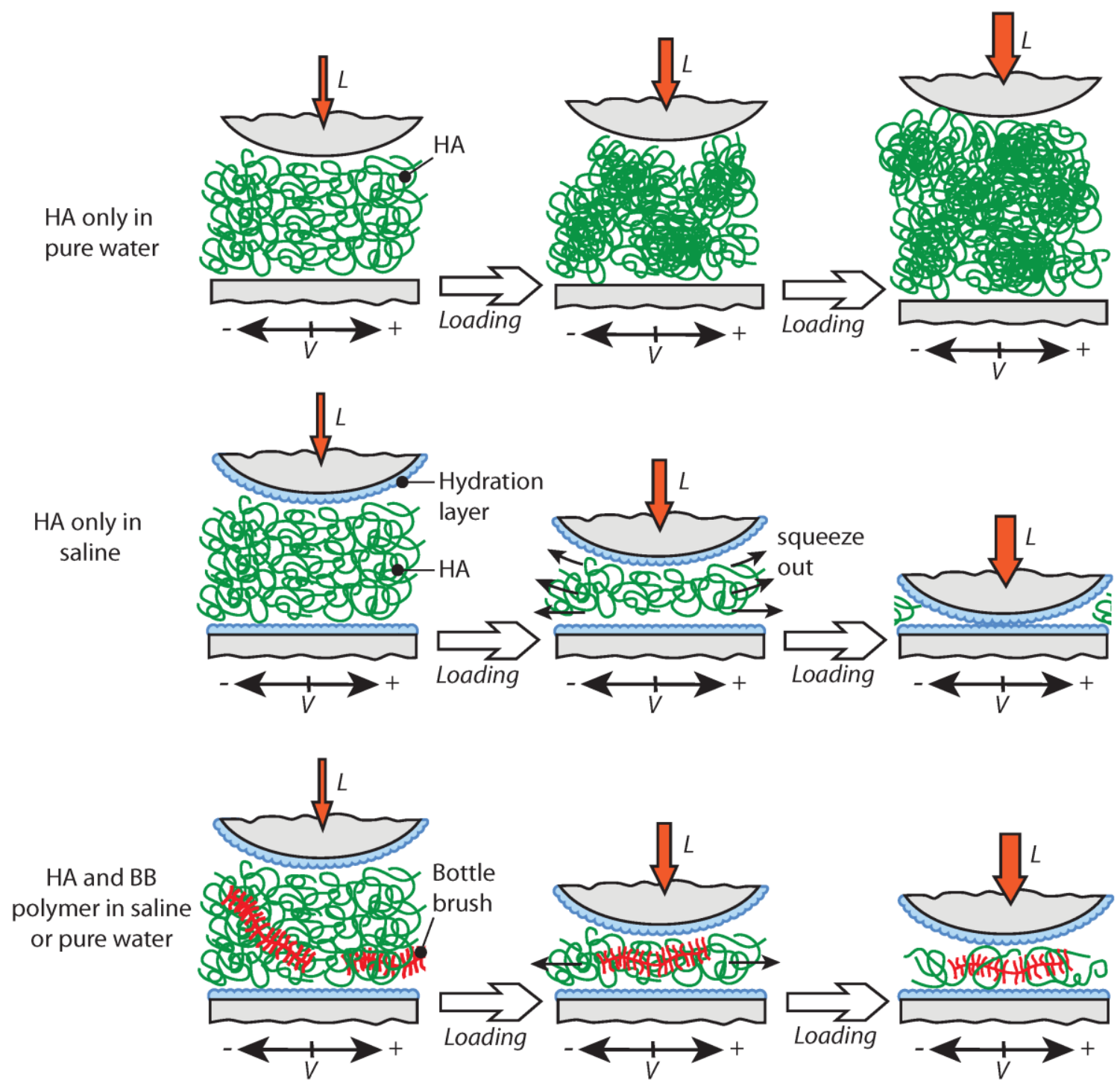

Figure 5. Schematic representation of the wear protection mechanism observed in presence of the mixture of $\mathrm{HA}$ and $\mathrm{BB}$ polymers in pure water and in saline.

In order to demonstrate the generality of the mechanism and its broad application, we performed a second series of tribological tests to establish the impact of shearing speed, BB:HA polymer ratio, polymer chemical structure and surface chemistry (see Figure 6). As shown in figure 6A, 
varying the sliding speed over three decades, between 0.01 and $10 \mu \mathrm{m} / \mathrm{s}$ at $P<P^{*}$, did not trigger any damage of the surfaces indicating a weak dependence, if any, of $P^{*}$ on the sliding speed. In figure $6 \mathrm{~B}$, we show that $P^{*}$ depends strongly on BB:HA ratio and is optimum at a ratio of $\mathrm{BB}: \mathrm{HA}=1: 10(\mathrm{mg} / \mathrm{mg})$. Above this optimum ratio, the value of $P^{*}$ is equal to the value of HA alone indicating that $\mathrm{HA}$ has displaced the $\mathrm{BB}$ polymer from the surface. Below the optimal ratio, the value of $P^{*}$ is equal to the value obtained for $\mathrm{BB}$ alone indicating that $\mathrm{BB}$ polymer is the sole component in the confined film. A similar synergistic behavior between BB polymer and HA was also observed when replacing HA by poly(N-vinylpyrrolidone) (PVP), a neutral, water soluble polymer $\left(M_{\mathrm{w}}=40 \mathrm{kDa}\right)$. Figure $6 \mathrm{C}$ shows that the value of $P^{*}$ in saline $(150 \mathrm{mM}, \mathrm{pH}=$ 7.4) exhibits a 2 fold increase with a 1:10 mixture ratio of BB:PVP compared to PVP alone. Similarly to HA, PVP did not show any direct interaction with the BB polymer by ITC (Figure S6). The HA - BB polymer mixture was tested against mica-gold tribo-pair as well. Gold being a ductile metal, its tribological properties are very poor in terms of wear resistance (Figure 6D). For the tribo-pair mica / gold, $P^{*}$ was inferior to $1 \mathrm{MPa}$ in presence of HA or BB polymer alone. As shown in Figure 6D, the polymer mixture was once again significantly more efficient in protecting the surfaces compared to the single components alone.

In all the tested conditions, the value of $P^{*}$ associated to the polymer mixture is systematically superior to the sum of the value associated to the polymers alone indicating a true synergistic interaction between both components in terms of wear protection.
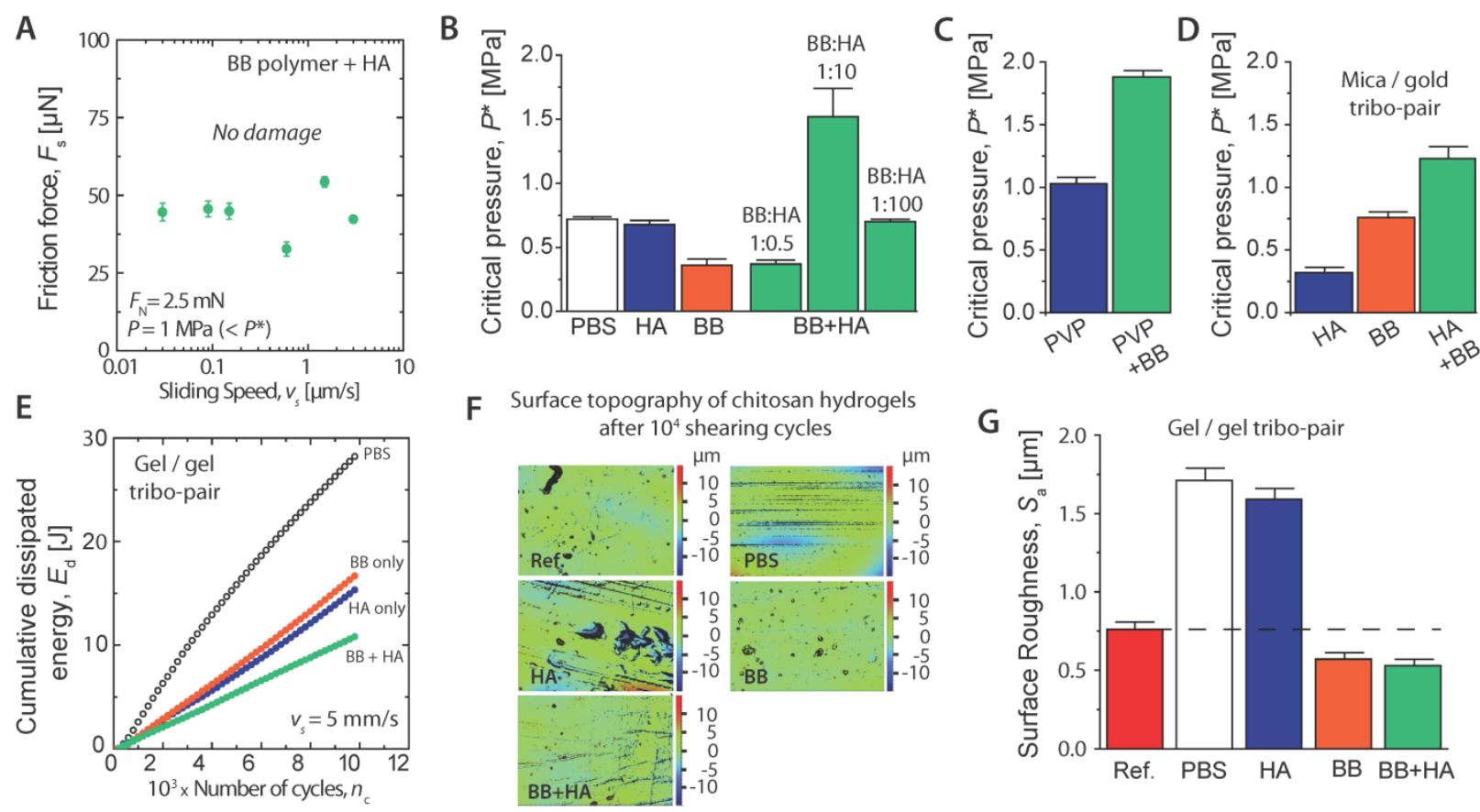

Figure 6. A) The wear protection imparted by the BB polymer - HA mixture was not affected by the sliding speed of the surfaces. At a pressure of $0.5 P^{*}$, no damage of the surfaces was observed even when varying the sliding speed by three orders of magnitude; B) The BB:HA (1.5 
MDa) ratio has a significant impact on $P^{*}$ and was found to be optimum at 1:10; C) HA can be replaced by PVP to obtain similar synergistic wear protection when mixed with the BB polymer; D) The BB polymer - HA mixture was tested with mica-gold tribo-pair demonstrating similar wear protection enhancement compared to mica-mica. E) Cumulative dissipated energy generated during shearing of two $2.5 \mathrm{w} \%$ chitosan hydrogel plugs, lubricated with different polymer solutions. F and G) Interferometric micrographs and associated surface roughness of the hydrogel plugs after $10^{4}$ shearing cycles at $5 \mathrm{~mm} / \mathrm{s}$ (shearing amplitude of $5 \mathrm{~mm}$, applied pressure $P=50 \mathrm{kPa}$ ).

We finally tested the lubricating fluids between macroscopic hydrogel plugs of chitosan as model soft polymeric surfaces (Figure 6E-G). Chitosan hydrogels have been extensively tested as cellular scaffolds for tissue engineering applications but their poor resistance against abrasive wear has hampered their translation to clinical settings. Tribo-testing of the hydrogels (2.5 w\%) have shown that the cumulated dissipated energy, $E_{\mathrm{d}}$, which is directly related to the wear volume, $W_{\mathrm{v}}{ }^{28}$ is strongly diminished in presence of the HA - BB mixture compared to each component alone. Concomitantly, the surfaces' roughness, $S_{\mathrm{a}}$, was found to significantly decrease in presence of the mixture compared to all other conditions due to surface polishing and restructuring.

The results shown in this study allow to confidently anticipate a large range of applications for such fluids. The polymers tested are known to be biocompatible and suitable for intra-articular injection which suggests a potential use as biolubricants. Other applications requiring wear protection of soft or ductile materials such as plastics (e.g. medical devices) or metals (e.g. articular implants) can be envisioned as well. The present strategy should be applicable to nonaqueous systems as well by simply adapting the chemical functionalities of the BB polymer and the linear polymer counterpart. On a more fundamental level, the lubricating system described in this study demonstrates that it is possible to control lubrication (i.e. the coefficient of friction $\mu$ ) and wear protection $\left(P^{*}\right)$ independently by tuning the properties of each component.

\section{Methods.}

BB polymer synthesis. Complete description of the polymer synthesis and characterization is provided in the suplementary information.

Surface Forces Measurements. Formulation of polymer mixtures: $10.0 \mathrm{mg}$ of different molecular weight HA were dissolved with magnetic stirring in $10 \mathrm{~mL}$ Milli-Q water or $10 \mathrm{mM}$ PBS pH 7.4 in a glass vial. The solution was kept at $4^{\circ} \mathrm{C}$ for $24 \mathrm{~h}$ prior to use. $1 \mathrm{mg} / \mathrm{mL}$ solution of BB polymer was prepared in the same buffers. $50 \mu \mathrm{L}$ of the polymeric solution was added to $450 \mu \mathrm{L}$ of HA solution corresponding to a solution of BB polymer at $100 \mu \mathrm{g} / \mathrm{mL}$ and $\mathrm{HA}$ at 0.9 $\mathrm{mg} / \mathrm{mL}$ and was homogenized with a vortex for $1 \mathrm{~min}$. The solution was centrifuged at 14,000 rpm during $10 \mathrm{~min}$ to remove aggregates or particles if any. For each SFA analysis, $50 \mu \mathrm{L}$ of fluid was injected between the surfaces. Surfaces were then let to equilibrate for $1 \mathrm{~h}$ prior to the measurements. 
Normal Interaction Forces: Measurements of the normal interaction forces between two opposing surfaces as a function of the separation distance were carried out using a Surface Forces Apparatus. The normal interaction force $F_{\mathrm{N}}$ is determined by measuring the deflection of the spring cantilever (spring constant of $482 \mathrm{~N} / \mathrm{m}$ ) supported by the lower surface. The distance between the surfaces is measured using Multiple Beam Interferometry. The two disks were mounted in the SFA chamber in cross cylinder geometry and brought into mica-mica adhesive contact in dry air in order to determine the reference position. Afterward, the cylindrical disks were separated by roughly $1 \mathrm{~mm}$ and the polymer solution was injected between the surfaces. Immediately after injection, the bottom of the SFA chamber was filled with water in order to saturate the surrounding vapors and to limit evaporation of the injected liquid. The normal interaction forces between the two polymer coated surfaces as a function of surface separation were determined on approaching (compression) and separating (decompression) the surfaces. For each test, all force runs (in and out) were performed at least in triplicate with the motor or the piezoelectric tube at a speed range of 0.4 to $1.6 \mathrm{~nm} / \mathrm{s}$. Each experiments were reproduced two to six times.

Friction Force Measurements: The friction force $F_{\mathrm{s}}$ was measured by moving the lower surface horizontally and measured the response of the upper surface. Before measuring the friction forces, three cycles of normal compression/decompression were performed on the same contact position. For friction tests, a piezo bimorph drove the lower surface in a back and forth motion at a constant sliding frequency of $50 \mathrm{mHz}$ controlled by a function generator. The friction force transmitted to the upper surface was detected by semi conductive strain gauges, and digitally recorded. Acquired data were processed using Origin ${ }^{\circledR}$ software. Separation distance and surface deformation were continuously recorded during the experiment using the FECO fringes analysis. Chitosan gels and tribotesting. A $2.5 \% \mathrm{w} / \mathrm{w}$ chitosan solution $\left(M_{\mathrm{w}} 6.04 .10^{5}, \mathrm{Mw} / \mathrm{Mn} 1.64\right.$, DA $4.3 \%$ ) was prepared by dissolving the polymer in an aqueous acetic acid solution. Air bubbles were removed by centrifugation and the highly viscous solution was compression-molded to obtain a slab of constant thickness. The chitosan solution was then placed in a $1 \mathrm{M} \mathrm{NaOH}$ coagulation bath to complete gelation. Gel disks of 11 and $21 \mathrm{~mm}$ in diameter were obtained using biopsy punchers and neutralized in pure water until use. For the tribo-testing experiments, the $11 \mathrm{~mm}$ diameter gel disk was glued on the top mobile part of a custom-made tribometer. The larger gel disk was glued on a metallic immobile bath filled with the tested polymer solution and left to incubate for $1 \mathrm{~h}$ prior to experimentation. Normal and tangential forces were recorded and analyzed with a home-made routine programmed in labview ${ }^{\circledR}$. Roughness of the gels was quantified after performing tribo-testing using an interferometric microscope.

\section{Acknowledgements.}

XB acknowledges the financial support from CIHR (950-228948 and ONM-143062) and NSERC (RGPIN-2014-06316). JF is grateful to the Arthritis Society (Award TGP-16-183) and the French Embassy (Frontenac) for financial supports. BRS thanks the financial support of GRUM. We are also indebted to Matthieu Guibert (LTDS) for the tribometer design and fabrication and Thomas Malhomme (LTDS) for wear assessment of the gels. KM and GX acknowledge support from NSF (DMR 1436219).

\section{Author contribution.}


JF and BRS performed SFA experiments, JB and GX synthesized the polymers. KM, JB and XB designed the BB polymer. SB, BRS and JF, performed tribological work. XB and JF wrote the manuscript. All the authors participated in the discussion of the data and in the production of the final version of the manuscript.

\section{Supporting Information.}

Synthesis of the Bottle-brush polymer

Interaction forces in presence of HA only

Interaction forces in presence of $\mathrm{HA}$ and $\mathrm{BB}$ polymer

Evolution of the refractive index and separation distance of confined saline and HA solution

during shear

\section{References}

1. Holmberg, K.; Andersson, P.; Erdemir, A. Global Energy Consumption Due to Friction in Passenger Cars. Tribol. Int. 2012, 47, 221-234.

2. Tzanakis, I.; Hadfield, M.; Thomas, B.; Noya, S. M.; Henshaw, I.; Austen, S. Future Perspectives on Sustainable Tribology. Renewable Sustainable Energy Rev. 2012, 16, 4126-4140.

3. Moro, T.; Takatori, Y.; Ishihara, K.; Konno, T.; Takigawa, Y.; Matsushita, T.; Chung, U.-i.; Nakamura, K.; Kawaguchi, H. Surface Grafting of Artificial Joints with a Biocompatible Polymer for Preventing Periprosthetic Osteolysis. Nat. Mater. 2004, 3, 829-836.

4. Klein, J. Chemistry. Repair or Replacement--a Joint Perspective. Science 2009, 323, 47-8.

5. Dedinaite, A. Biomimetic Lubrication. Soft Matter 2012, 8, 273-284.

6. Schmidt, T. A.; Gastelum, N. S.; Nguyen, Q. T.; Schumacher, B. L.; Sah, R. L. Boundary Lubrication of Articular Cartilage - Role of Synovial Fluid Constituents. Arthritis Rheum. 2007, 56, 882-891.

7. Liu, X.; Dedinaite, A.; Rutland, M.; Thormann, E.; Visnevskij, C.; Makuska, R.; Claesson, P. M. Electrostatically Anchored Branched Brush Layers. Langmuir 2012, 28, 15537-47.

8. Pettersson, T.; Naderi, A.; Makuska, R.; Claesson, P. M. Lubrication Properties of Bottle-Brush Polyelectrolytes: An Afm Study on the Effect of Side Chain and Charge Density. Langmuir 2008, 24, 3336-3347.

9. Liu, X.; Thormann, E.; Dedinaite, A.; Rutland, M.; Visnevskij, C.; Makuska, R.; Claesson, P. M. Low Friction and High Load Bearing Capacity Layers Formed by Cationic-Block-Non-Ionic Bottle-Brush Copolymers in Aqueous Media. Soft Matter 2013, 9, 5361-5371.

10. Chen, M.; Briscoe, W. H.; Armes, S. P.; Cohen, H.; Klein, J. Polyzwitterionic Brushes: Extreme Lubrication by Design. Eur. Polym. J. 2011, 47, 511-523.

11. Ohsedo, Y.; Takashina, R.; Gong, J. P.; Osada, Y. Surface Friction of Hydrogels with Well-Defined Polyelectrolyte Brushes. Langmuir 2004, 20, 6549-6555.

12. Raviv, U.; Giasson, S.; Kampf, N.; Gohy, J. F.; Jerome, R.; Klein, J. Lubrication by Charged Polymers. Nature 2003, 425, 163-165.

13. Tairy, O.; Kampf, N.; Driver, M. J.; Armes, S. P.; Klein, J. Dense, Highly Hydrated Polymer Brushes Via Modified Atom-Transfer-Radical-Polymerization: Structure, Surface Interactions, and Frictional Dissipation. Macromolecules 2015, 48, 140-151.

14. Kobayashi, M.; Tanaka, H.; Minn, M.; Sugimura, J.; Takahara, A. Interferometry Study of Aqueous Lubrication on the Surface of Polyelectrolyte Brush. ACS Appl. Mater. Interfaces 2014, 6, 20365-20371. 
15. Morse, A. J.; Edmondson, S.; Dupin, D.; Armes, S. P.; Zhang, Z.; Leggett, G. J.; Thompson, R. L.; Lewis, A. L. Biocompatible Polymer Brushes Grown from Model Quartz Fibres: Synthesis, Characterisation and in Situ Determination of Frictional Coefficient. Soft Matter 2010, 6, 1571-1579.

16. Klein, J.; Kumacheva, E.; Mahalu, D.; Perahia, D.; Fetters, L. J. Reduction of Frictional Forces between Solid-Surfaces Bearing Polymer Brushes. Nature 1994, 370, 634-636.

17. Klein, J. Shear, Friction, and Lubrication Forces between Polymer-Bearing Surfaces. Annu. Rev. Mater. Sci. 1996, 26, 581-612.

18. Banquy, X.; Burdynska, J.; Lee, D. W.; Matyjaszewski, K.; Israelachvili, J. Bioinspired Bottle-Brush Polymer Exhibits Low Friction and Amontons-Like Behavior. J. Am. Chem. S.2014, 136, 6199-6202.

19. Sheiko, S. S.; Sumerlin, B. S.; Matyjaszewski, K. Cylindrical Molecular Brushes: Synthesis, Characterization, and Properties. Prog. Polym. Sci. 2008, 33, 759-785.

20. Kobayashi, M.; Terayama, Y.; Kikuchi, M.; Takahara, A. Chain Dimensions and Surface Characterization of Superhydrophilic Polymer Brushes with Zwitterion Side Groups. Soft matter 2013, 9 , 5138-5148.

21. Chen, M.; Briscoe, W. H.; Armes, S. P.; Klein, J. Lubrication at Physiological Pressures by Polyzwitterionic Brushes. Science 2009, 323, 1698-1701.

22. Maier, G. P.; Rapp, M. V.; Waite, J. H.; Israelachvili, J. N.; Butler, A. Biological Adhesives. Adaptive Synergy between Catechol and Lysine Promotes Wet Adhesion by Surface Salt Displacement. Science 2015, 349, 628-32.

23. Petrone, L.; Kumar, A.; Sutanto, C. N.; Patil, N. J.; Kannan, S.; Palaniappan, A.; Amini, S.; Zappone, B.; Verma, C.; Miserez, A. Mussel Adhesion Is Dictated by Time-Regulated Secretion and Molecular Conformation of Mussel Adhesive Proteins. Nat. Commun. 2015, 6.

24. Ma, L.; Gaisinskaya-Kipnis, A.; Kampf, N.; Klein, J. Origins of Hydration Lubrication. Nat. Commun. 2015, 6, 6060.

25. Heuberger, M.; Luengo, G.; Israelachvili, J. Topographic Information from Multiple Beam Interferometry in the Surface Forces Apparatus. Langmuir 1997, 13, 3839-3848.

26. Perkin, S.; Goldberg, R.; Chai, L.; Kampf, N.; Klein, J. Dynamic Properties of Confined Hydration Layers. Faraday Discuss. 2009, 141, 399-413; discussion 443-65.

27. Raviv, U.; Perkin, S.; Laurat, P.; Klein, J. Fluidity of Water Confined Down to Subnanometer Films. Langmuir 2004, 20, 5322-32.

28. Fouvry, S.; Liskiewicz, T.; Kapsa, P.; Hannel, S.; Sauger, E. An Energy Description of Wear Mechanisms and Its Applications to Oscillating Sliding Contacts. Wear 2003, 255, 287-298. 
Table of Contents.

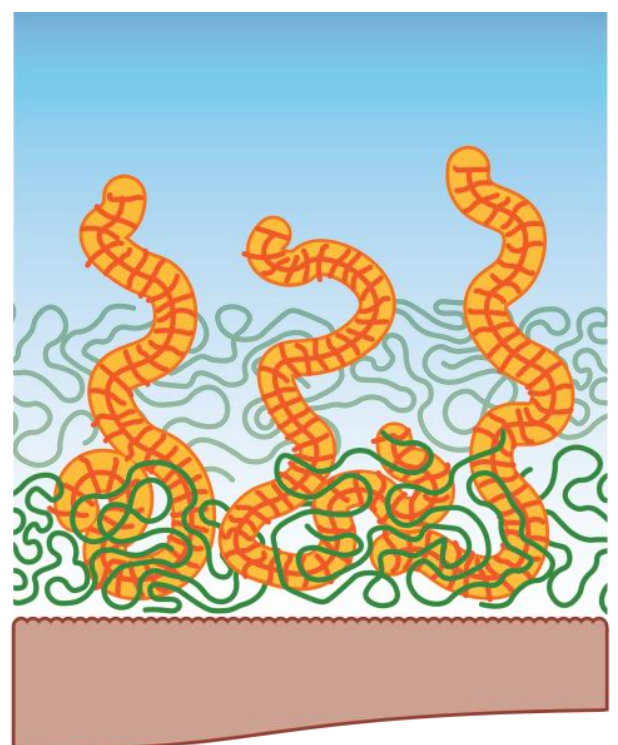

